\title{
Molecular dynamic and kinetic characterization of Cas9 mutants for improving specificity of genome editing tools
}

\author{
Vokhtantsev I. ${ }^{1,2 *}$, Kadtsyn E. ${ }^{2,3}$ \\ ${ }^{1}$ Institute of Chemical Biology and Fundamental Medicine SB RAS, Novosibirsk, Russia \\ ${ }^{2}$ Novosibirsk State University, Novosibirsk, Russia \\ ${ }^{3}$ Voevodsky Institute of Chemical Kinetics and Combustion SB RAS, Novosibirsk, Russia \\ *e-mail: ivanvohtancev@gmail.com
}

Key words: CRISPR/Cas9, molecular dynamics, genome editing

Motivation and Aim: Rational design was widely used to improve Cas9 genome editing tools in the last decade. In this approach amino acids, involved in stabilization of non-target strand DNA or destabilization of sgRNA/DNA, was mutated to increase a specificity of CRISPR/Cas9 system. Molecular dynamics (MD) data can elucidate thermodynamic aspects of interaction between amino acids and heteroduplex. We decided to find mutants that destabilize heteroduplex and then characterize them using MD and cleavage kinetics measurements to obtain more specific Cas9 variants.

Methods and Algorithms: The structure of the Cas9/sgRNA/DNA complex from [2] was used for molecular dynamics. The simulation was carried out in the Gromacs 5.1.2 package, at 300K and 1 bar. Force fields were used: ff14sb for protein, OL15 for DNA, OL3 for RNA, for water tip3p model was chosen, the charge was neutralized with sodium ions. The MD trajectory length was $100 \mathrm{~ns}$; a $30 \mathrm{~ns}$ segment was used for analysis. The energies of interaction of individual amino acids with the sgRNA/DNA heteroduplex were estimated within the APBS approach using the g_mmpbsa program. Recombinant Cas9 protein, sgRNA, and radiolabeled duplexes with/without mismatch $(4,9,14,18 \mathrm{bp}$ mismatch) were obtained for kinetic characterization. Single-turnover kinetics was carried out with 40 -fold excess over substrate by duplex addition. Cas9 point mutants have also been simulated (K163A, F164A, R403A, K918A) and characterized in the same conditions.

Results: After first round MD simulation, amino acids (K163, F164, R403, K918) with different energies of interaction were chosen for substitution into alanine and further simulation. As a result, the total energy values for all amino acids were compared for the native protein and mutants. It can be concluded that the first mutation stabilizes the heteroduplex in the protein, the rest destabilize, and the greatest effect is achieved for F164A. Kinetic studies have shown that there are no significant changes in the observed constants for the wild-type and mutant proteins when the substrate without mismatches was cleaved. For all mismatches, there was a decrease in wild-type cleavage activity. Specificity profiles of K163A and F164A mutants were slightly different between each other and between wild type protein. Interestingly, R403A mutant was more specific than the wild-type protein, but K918A was less specific.

Conclusion: We obtain a more specific Cas9 R403A mutant, and less specific Cas9 K918A mutant, but MD data not clearly correlate with kinetics. As a perspective we model heteroduplex with mismatch to find thermodynamics basics of Cas9 specificity.

\section{References}

1. Zuo Z, Liu J. Cas9-catalyzed DNA Cleavage Generates Staggered Ends: Evidence from Molecular Dynamics Simulations. Sci Rep. 2016;5:37584. 